\title{
Editor's Message
}

This is the first of a few special editions that the Environmental Health Review has planned over the next couple of years. In this issue, we present a few evidence reviews. One of the main functions of Environmental Health Review is to increase the amount of evidence available to environmental public health professionals. Further, moving the Environmental Health Review to a peer-reviewed format has increased the amount of high-quality information available that can be used toward evidence informed decision making.

These evidence reviews are broad ranging and are meant to provide a snapshot of the current evidence of a subject matter. This work builds on the already robust catalog of evidence reviews produced by the National Collaborating Center for

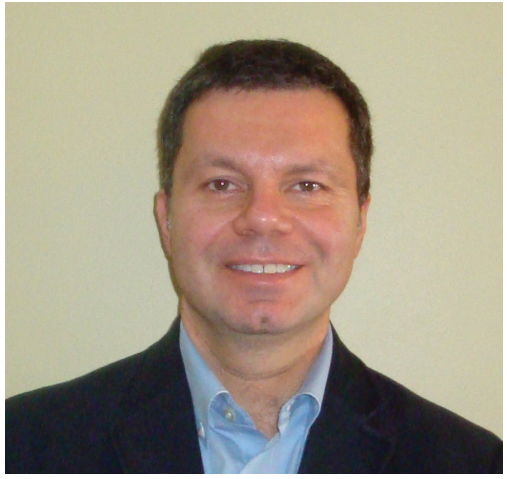

Environmental Health. Information presented in this issue does not in and of itself provide direction, but it hopefully provides guidance and a base level of information on the subject. Ideally this information is then discussed and debated among environmental public health professionals, and the consensus direction is derived through these deliberations.

I hope you enjoy reading these evidence reviews and more importantly become better informed on emerging environmental public health matters. Please stay tuned for another special edition before the end of 2014 .

Andrew Papadopoulos Editor-in-Chief 